\title{
Validation of the Model Obtained Ice Drift Fields Based on Satellite Derived Data Using a Vector Correlation Indexes in an Invariant Form
}

\author{
Vladimir Aleksandrovich Volkov ${ }^{1}$, Denis Mikhailovich Demchev ${ }^{1,2}$ and Nikolay Evgenievich Ivanov ${ }^{2}$ \\ 1. Nansen International Environmental and Remote Sensing Center (NIERSC), Applied Meteorological and Oceanographic \\ Research Group, St. Petersburg 199034, Russia
}

2. Arctic and Antarctic Research Institute (AARI), Center for Ice and Hydrometeorogical Information, St. Petersburg 199397, Russia

\begin{abstract}
The article considers results of validation of a sea ice modeling data using a novel methodology on the vector algebra theoretical bases. The vectorial-algebraic approach developed and was in use in Russia for an analysis of time series of vector values - wind, currents ice drift etc. The vectorial-algebraic approach allows significantly compressing the initial information and most adequately describes the vector time series of full-scale and model data restricted by a set of statistical characteristics in the invariant form. For an express analysis of correlation of the modeling data and in situ (or satellite derived) data a system of simplified correlation invariant indicators was used. This methodology was applied for validation of vector values fields at the first time. The method gives an opportunity to describe a field of a vector correlation and detect an areas with different levels of correlation between model and in situ (or other reference) vector data. The work was carried out in the frame of the My Ocean Project (FP7).
\end{abstract}

Key words: Sea ice, vector correlation, model validation, ice drift.

\section{Introduction}

The authors of this article faced the problem of validation of the model sea ice drift fields and analysis of a large amount of information on the vector fields in the My Ocean Project (FP7) and attempted to adapt the so-called vectorial-algebraic approach [1] to the project objectives.

Since the ice drift (similar to currents and wind) is often considered as a vector probabilistic process and is described by a set of two-dimensional vectors with a specific time step, the choice of the mathematical vector model is the basic problem in selecting a methodology for analyzing the ice drift or other vector series.

The applied studies often use a vector presentation in the form of components - projections to the orthogonal

Corresponding author: Dr. Vladimir Volkov, Ph.D., research director/head of MetOcean group, research fields: polar oceanography, climate variability, model validation. axes, as a complex number, and in the form of the motion vector, which has a module (vector magnitude) and a direction, which is added with similar vectors by the parallelogram rule. Since the drift (or a current/wind) is a progressive motion of ice (water, air) masses, an approach to the analysis of such processes through a displacement vector and the analysis method based on the vector algebra laws turns out to be most valid. Not dwelling in detail on comparison of the aforementioned approaches in vector presentation, we will refer to Ref. [2]. This publication shows that using a component-wise, complex-valued presentation of vectors or applying a method of rotational components for the analysis of vector processes, it is possible to obtain an illusionary, non-adequate understanding about the investigated natural process. Only the vectorial-algebraic method yields physically-substantiated conclusions in the analysis of full-scale data, because it strictly takes into account the vector character of the process and presents the results 

Data Using a Vector Correlation Indexes in an Invariant Form

of time series analysis by a compact set of scalar characteristics in the invariant form, i.e., not dependent on the choice of the coordinate system.

In the validation aspect the method gives an opportunity to detect in the model areas a geographical region with different levels of correlation between model and ground-based measurements (in situ data), as well as to compare some temporal structure of variability in different geographical regions. As a reference ground-based measurements the data about the drift, computed based on satellite information were used (http://iabp.apl.washington.edu, http://cersat.ifremer.fr/data/discovery/by_product_typ e/gridded_products/psi_amsr_drift,

$\mathrm{http}$ ://cersat.ifremer.fr/science/sea_ice/validation_of_a rctic_sea_ice_drift_with_iabp_buoys, Fowler, C. 2003, updated 2007. Polar Pathfinder Daily $25 \mathrm{~km}$ EASE-Grid Sea Ice Motion Vectors. Boulder, Colorado USA: National Snow and Ice Data Center. Digital Media). It should be noted that the bases of the vectorial-algebraic approach to the analysis of vector series were developed quite a long time ago - from the $80 \mathrm{~s}$ of the last century, but so far this method is used infrequently. This method has however a status of a standard method in the framework of the Russian uniform information system of the world ocean-ESIMO (http://www.esimo.ru). In the opinion of the authors, the method is little known outside Russia. More widespread method by Crosby et al. [3], but it is a slightly different approach to the problem of the vector correlation.

\section{Data Used}

As reference measurements, the data about the ice drift fields the low resolution ice drift dataset retrieved from AMSR-E were used. The vector drift fields derived by processing the AMSR-E sensor data are presented at regular grid points with a step of $31.25 \mathrm{~km}$ over the entire Arctic Ocean area, where sea ice was present in the wintertime. These data with a 2-day time interval prepared by the Center for Satellite
Exploitation and Research (CERSAT) at IFREMER (France) are available for the period 1 October 2002 through present and can be accessed via the Internet. Estimates of the accuracy of such fields performed by developers show this data set to allow an adequate assessment of ice dynamics in the Arctic Basin. It is a merging of the sea-ice drift vectors retrieved separately using respectively vertically and horizontally polarized $89 \mathrm{GHz}$ brightness temperature maps (since winter of 1991-1992). The estimation of sea-ice drift for each one relies on a basic cross correlation technique applied to the field of the second spatial derivative (Laplacian) of $6.25 \mathrm{~km}$ resolution composite maps separated by a few days. A preliminary coarse drift validation is performed using an interpolated ECMWF wind field averaged over either two, three or six days. Then local consistency controls are performed using neighbouring drift vectors. These intermediate results are saved and used for producing the final merged AMSR drift.

As a model sea ice drift data for demonstration of possibilities of the vectorial-algebraic approach for validation a limited set of test data from the TOPAZ4 modelling system, two models run with and without data assimilation. The complete description of the modeling and data assimilation system can be found by Sakov et al. [4] but the essential elements will be reminded here. TOPAZ4 uses the HYCOM model [5] in its version 2.2 in a north atlantic and Arctic basins configuration, coupled to an EVP (elastic-visco-plastic) sea ice model and forced by the ECMWF ERA-interim reanalysis fields. The model horizontal resolution is about $12 \mathrm{~km}$ in the whole Arctic. In data assimilation mode, 100 dynamical members are running forward in time and several data types are assimilated using the deterministic ensemble Kalman Filter. Those are satellite data of sea level anomalies, sea surface temperatures, temperature and salinity profiles from the Argo buoys and the IPY DAMOCLES ice-tethered profilers, sea ice concentrations from the OSI-SAF service at met.no and the sea ice drift products described above. The free run is conducted in the same 
conditions but without any data assimilation.

The assimilation of ice concentrations effectively corrects the position of the ice edge, as noted by Lisaeter et al. [6]. In contrast, the assimilation of ice drift has surprisingly little effect-two orders of magnitude less efficient than with ice concentrations - which may be explained by a lack of degrees of freedom in the sea ice rheology as the EVP model is known to under-represent the randomness of ice drift [7], the effect is however evenly distributed in the interior of the ice pack.

The model output is produced in daily averages and the ice drift vectors rotated according to the output grid. Monthly averages of the TOPAZ4 data are available from the My Ocean data server ${ }^{(\mathrm{e})}$.

\section{Methodology of Analysis}

Since wind, sea currents and the ice drift present a directed mass transfer, their speeds are physical vectors. Therefore, we assume the Euclidean vector $\vec{V}$ with Cartesian projections $V_{x}, V_{y}$ has been assumed as a speed model — a mathematical object characterized by module $|\vec{V}|$ and direction $\varphi$, for which we have determined an addition by the rule of parallelogram, rules of transformation of vector components at transfer to a new coordinate system and three types of vector multiplying — scalar, vector (oblique) and tensor [8]. The vectorial-algebraic method based on these provisions [1] allows us to consider data in the terms of a vector random value, a vector random process, a system of connected vector random values and a vector spatial-temporal random field.

In the frame of the vectorial-algebraic approach for describing of the distributions of probabilities, one usually uses the distribution moments, being restricted most often by the first initial moment-mathematical expectation and the second central moment-dispersion. The mathematical expectation is determined as vector $\vec{m}_{\vec{V}}$, its projections being equal to mathematical expectations $\left(m_{V_{X}}, m_{V_{y}}\right)$ of projections of vector $\vec{V}$ are

$$
\vec{m}_{\vec{V}}=M\left\{V_{X} \vec{e}_{X}+V_{Y} \vec{e}_{Y}\right\}
$$

where, $\vec{e}_{X}, \vec{e}_{Y}$-orts of axes OX, OY. This determination coincides with the determination of mean speed in the component-wise method, in which the speed model is the matrix-line $\left(m_{V_{X}}, m_{V_{y}}\right)$ or a corresponding matrix-column.

The correlation function in the stationary approximation $K_{\vec{V}}(\tau)$ is determined as a mathematical expectation $\mathrm{M}$ of tensor product $\otimes$ of centered vectors $\vec{V}^{0}=\vec{V}-\vec{m}_{\vec{V}}$, displaced over the time interval $\tau$

$$
K_{\vec{V}}(\tau)=M\left\{\vec{V}^{0}(t) \otimes \vec{V}^{0}(t+\tau)\right.
$$

Function $K_{\vec{V}}(\tau)$ characterizes the interrelation of the directed speed changes at the moments of time shift by interval and gives a quantitative measure of their intensity and orientation in the prescribed coordinate system.

For flat vector $\vec{V}$ at the fixed time shift, $K_{\vec{V}}(\tau)$ is tensor of the second rank the elements of which are represented by co-variations of the corresponding projections. This tensor can be expanded in the only way to a sum of symmetric and skew-symmetric tensors

$$
\begin{aligned}
K_{\vec{V}}(\tau)= & \left(\begin{array}{cc}
K_{V_{x} V_{x}}(\tau) & K_{V_{x} V_{y}}(\tau) \\
K_{V_{y} V_{x}}(\tau) & K_{V_{y} V_{y}}(\tau)
\end{array}\right)=C(\tau)+G(\tau)= \\
& \left(\begin{array}{cc}
\lambda_{1}(\tau) & 0 \\
0 & \lambda_{2}(\tau)
\end{array}\right)+0.5 G(\tau)\left(\begin{array}{cc}
0 & 1 \\
-1 & 0
\end{array}\right)
\end{aligned}
$$

The components of tensor $K_{\vec{V}}(\tau)$ depend in the general case on the choice of the coordinate system, however invariants exist-scalar functions of components that do not depend on the change of the coordinate system. Invariants $\lambda_{1,2}(\tau)$ of the symmetrical part $C$ are the values by each fixed $\tau$

$$
\lambda_{1,2}=0.5\left\{I_{1} \pm \sqrt{\left(K_{V_{x}}-K_{V_{y}}\right)^{2}+2 K_{V_{x} V_{y}}^{2}}\right\}
$$

and tensor $C$ itself is reduced to a symmetrical form by the initial coordinate system turning by an angle

$$
\alpha=0.5 \operatorname{arctg} \frac{2 K_{V_{x} V y}}{K_{V_{x}}-K_{V y}} \pm 180^{\circ}
$$

The skew-symmetrical tensor invariant

$$
G=K_{V_{x} V_{y}}-K_{V_{y} V_{x}}
$$


is called the rotation indicator, and the sum of $\lambda_{1,2}$ is called a linear invariant $I_{1}$

$$
I_{1}=\lambda_{1}+\lambda_{2}
$$

Since Eqs. (4)-(8) contain all elements of tensor $K_{\vec{V}}(\tau)$, they stress it unlike from the correlation matrix, each element of which has an independent value. The invariants themselves characterize different variability properties.

Dispersion is a co-variation function value at zero time shift $D_{\vec{V}} \equiv K_{\vec{V}}(0)$. Since the reciprocal co-variations of projections are in this case equal to $K_{V_{x} V_{y}}(0)=K_{V_{y} V_{x}}(0)$, the dispersion tensor $D_{\vec{V}}$ is symmetrical

$$
D_{\vec{V}}=\left(\begin{array}{cc}
D_{V_{x}} & K_{V_{x} V_{y}} \\
K_{V_{y} V_{x}} & D_{V_{y}}
\end{array}\right)=\left(\begin{array}{cc}
\lambda_{1} & 0 \\
0 & \lambda_{2}
\end{array}\right)
$$

And its linear invariant $I_{1}$ determines the general intensity of variability $\vec{V}$ due to changes of both the module and the direction.

The RMSD (root-mean-square deviation) tensor $\sigma_{\vec{V}}$ is determined as

$$
\sigma_{\vec{V}}=\sqrt{D_{\vec{V}}}=\left(\begin{array}{cc}
\lambda_{1}^{(\sigma)} & 0 \\
0 & \lambda_{2}^{(\sigma)}
\end{array}\right)
$$

Its invariants are the square roots of the corresponding invariants of tensor $D_{\vec{V}}-\lambda_{1,2}^{(\sigma)}=\lambda_{1,2}^{(D)}$, then we will use for tensor $\sigma_{\vec{V}}$ the designations $\lambda_{1,2}$ without the upper index. For characterization of the total variability (cumulative effect of module and direction variability $\vec{V}$ ), we will use $\sqrt{I_{1}^{(D)}}$, of tensor $D_{\vec{V}}$, designating it for homogeneity as $I_{1}^{(0.5)}$.

Geometrically (Fig. 1), $\sigma_{\vec{V}}$ is an ellipse with semi-axes $\lambda_{1,2}$ as Eq. (5), oriented in the maximum variability direction $\alpha$ shown in Eq. (5). This also underlines the advantage of the vectorial-algebraic method as compared with the component-wise method, in which the maximum variability direction can be determined only in the framework of the a-priori hypothesis on the form of distribution of projections (for example, a two-dimensional normal distribution). Graphically (both for the individual points, and on the charts) $\sigma_{\vec{V}}$ is represented by an ellipse and/or by ellipse axes, and it is useful to mach it with the mean speed vector $\vec{m}_{\vec{V}}$.

Such representation, which is an embodiment of the vectorial-algebraic approach provides for a very significant information compression. Instead of lengthy recurrence tables, the characteristics of variability of the time series of the vector process can be represented by only several numbers.

One often has to deal with vector systems $\{\vec{V}, \vec{U}\}$, the properties of which are not completed with the properties of individual components, but also include interdependencies, for example - with speeds and ice drift, drift speeds from full-scale and model data-a validation case or the drift speeds at several points.

The covariation of the system of connecting vector random values is represented by a tensor of cross-variance

$$
\begin{array}{r}
D_{\vec{V} \vec{U}}=\left(\begin{array}{ll}
K_{V_{x} U_{x}} & K_{V_{x} U_{y}} \\
K_{V_{y} U_{x}} & K_{V_{y} U_{y}}
\end{array}\right)= \\
\left(\begin{array}{cc}
\lambda_{1} & 0 \\
0 & \lambda_{2}
\end{array}\right)+0.5 G\left(\begin{array}{cc}
0 & 1 \\
-1 & 0
\end{array}\right)
\end{array}
$$

The kinematic treatment of its invariants is similar to that of tensor $K_{\vec{V}}(\tau \neq 0)$.

Obukhov [9] determined the linear regression of abstract algebraic vectors by equation

$$
\vec{V}_{i}=A_{\vec{V} \vec{U}} \vec{U}_{l}+\vec{B}_{\vec{V} \vec{U}}+\vec{\varepsilon}_{i} \equiv \widehat{\vec{V}}_{l}+\vec{\varepsilon}_{i}
$$

where, $A_{\vec{V} \vec{U}}=D_{\vec{V} \vec{U}} D_{\vec{V}}^{-1}$-regression tensor, $\vec{B}_{\vec{V} \vec{U}}=$ $\vec{m}_{\vec{V}}-A_{\vec{V} \vec{U}} \vec{m}_{\vec{U}}$-vector (analogues of coefficient $a$ and a free term $b$ of regression of scalar values $Y=a X=$ $b)$. Vector of unexplainable residual was predicted by regression in Eq. (11)- $\vec{\varepsilon}_{i}=\vec{V}_{i}-\widehat{\vec{V}}_{l}$. It is shown in Ref. [10] that Eq. (11) is also applicable to the case of Euclidian vectors.

In the component-wise model of vectors [11] determined the regression by a system of equations 


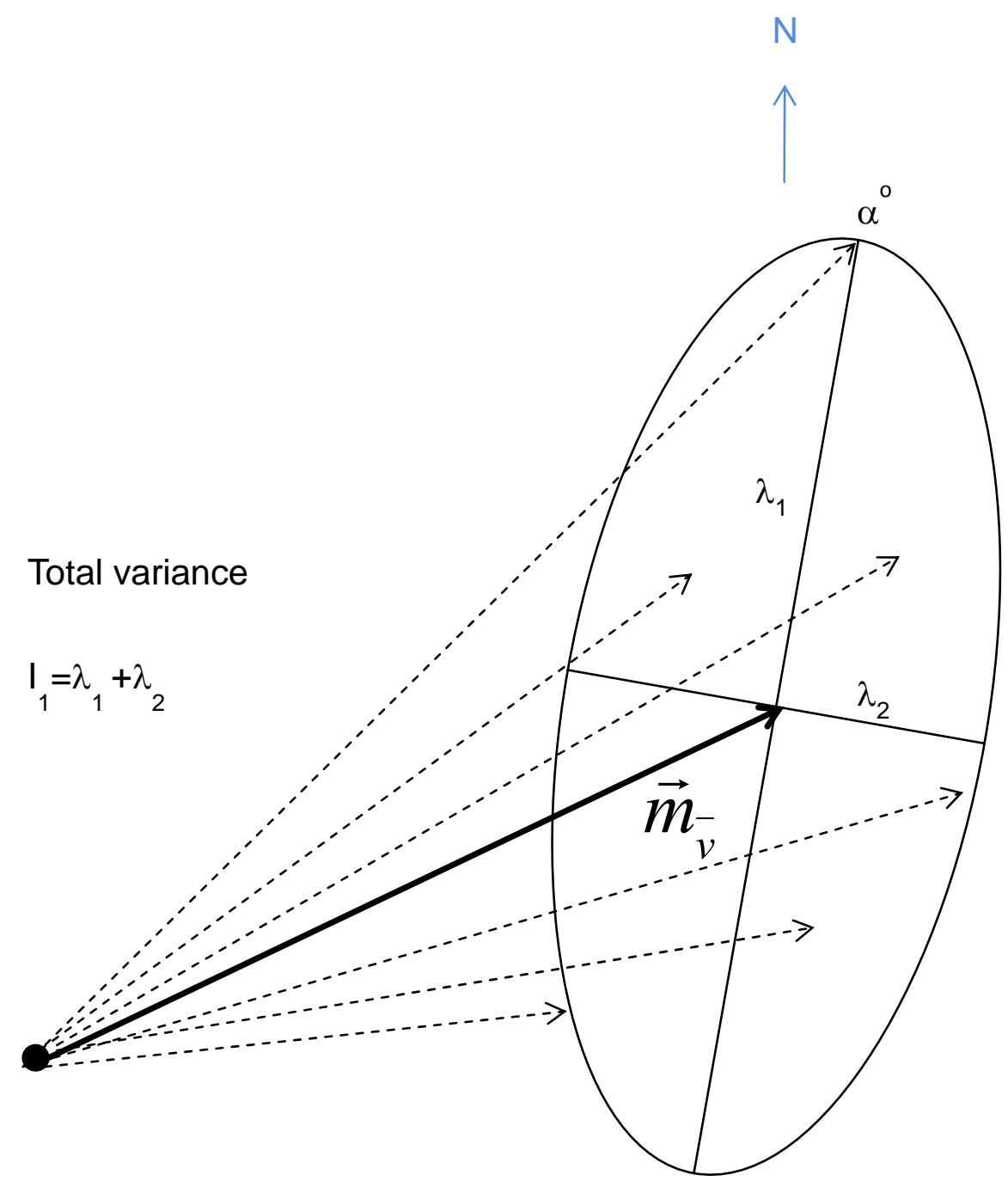

Fig. 1 Graphic interpretation of the variance tensor characteristics obtained making use of the vectorial-algebraical method. Source: $\vec{m}_{\vec{V}}$ : Vector of mathematical expectation — an average vector for a given realisation (absolute value and direction);

$\lambda_{1}, \lambda_{2}$ : The MSD (mean square difference) of the orthogonal components of the current velocity along the axes of maximum and minimum variability of the vector value - elements of the MSD ellipse;

$\alpha^{\text {o: }}$ : The direction of the major axis of the MSD ellipse;

$\mathrm{I}_{1}$ : Analogue to the MSD, a quantitative characteristic of the general variability of the vector process, independent of the fact whether the module or the wind (drift, current) direction changes;

Dotted vectors: Some vectors of the initial data set.

$$
\begin{aligned}
& V_{X}=a_{X} U_{X}+b_{X} U_{Y}+C_{X}+\varepsilon_{X} \\
& V_{Y}=a_{Y} U_{Y}+b_{Y} U_{Y}+C_{Y}+\varepsilon_{Y}
\end{aligned}
$$

the numerical solution of which is consistent with Eq. (11).

The linear regression closeness Eq. (11) is determined by correlation, which should take into account a combined effect of cross-changes of modules and directions of vectors $\vec{V}$ and $\vec{U}$. The component-wise regression model Eq. (12), in which correlation is represented by a matrix-line of coefficients of multiple linear regressions $\left\{R_{V_{x} ; U_{x} U_{y}} R_{V_{y} ; U_{x} U_{y}}\right\}$ complicates the interpretation, as a combined interpretation of the elements of this matrix is impossible without the additional assumptions. Since 
in the vectorial-algebraic method, covariation $D_{\vec{V} \vec{U}}$ in Eq. (10) and regression coefficient $A_{\vec{V} \vec{U}}$ in Eq. (11) are second rank tensors, the vector correlation should also be a second rank tensor and it is so determined in Ref. [9]

$$
R_{\vec{V} \vec{U}}^{2}=A_{\vec{V} \vec{U}} A_{\vec{U} \vec{V}}
$$

- Vector analogue of determination of the correlation coefficient of scalar values $r_{X Y}=a_{X Y}$. $\mathrm{a}_{\mathrm{YX}}$ and in Ref. [10]

$$
\widetilde{R}_{\vec{V} \vec{U}}=D_{\vec{V} \vec{U}} D_{\vec{V}}^{-0.5} D_{\vec{U}}^{-0.5}
$$

- vector analogue of determination of correlation coefficient $r_{x y}=D_{x y} D_{x}^{-0.5} D_{y}^{-0.5}$.

For linear independent vectors the tensors $R_{\vec{V} \vec{U}}^{2}, \widetilde{R}_{\vec{V} \vec{U}}$ are represented by zero matrixes and in the case of deterministic linear dependence - by single matrixes. However in the general case of stochastic dependence, their kinematic interpretation is difficult due to multidimensionality (22-23). That is why a system of simplified correlation indicators is proposed in Ref. [10] and described in Ref. [12], which are determined through invariants of the covariance tensor $D_{\vec{V} \vec{U}}$ and dispersion tensors $D_{\vec{V}}, D_{\vec{U}}$,

$$
r_{\uparrow \downarrow}=\frac{I_{1}^{(\overrightarrow{V U U})}}{\sqrt{I_{1}^{(\vec{V})} I_{1}^{(\vec{U})}}}, r_{\perp}=\frac{G_{1}^{(\vec{V} \vec{U})}}{\sqrt{I_{1}^{(\vec{V})} I_{1}^{(\vec{U})}}}, \mu=\sqrt{r_{\uparrow \downarrow}^{2}+r_{\perp}^{2}}
$$

represented by indicators of the collinear $r_{\uparrow \downarrow}$, orthogonal $r_{\perp}$ correlation and the indicator of vector correlation $\mu$. For linearly independent vectors $=0$. At deterministic linear dependence, the indicators $r_{\uparrow \downarrow}$, $r_{\perp}$ attain values from the interval of \pm 1 which provide fulfillment of equality $\mu=1$, and at the stochastic dependence-fulfillment of inequality $\mu<1$. If the collinear vector components $\vec{V}$ and $\vec{U}$ are mainly uniaxial, then $r_{\uparrow \downarrow}>0$ and vice versa. If the second vector $\vec{U}$ is turned predominantly to the right relative to the first vector $\vec{V}$, then $r_{\perp}>0$ and vice versa. If the angle between vectors $\vec{V}$ and $\vec{U}$ is equal to $45^{\circ}$, then $r_{\uparrow \downarrow}=r_{\perp}$ and $r_{\perp}$ by the module, if the angle is smaller than $45^{\circ}$, then the module $r_{\uparrow \downarrow}$ is larger than the module $r_{\perp}$ and vice versa. In the case of coincidence of $\vec{V}$ and $\vec{U}$

$$
r_{\uparrow \downarrow}=\mu=1, r_{\perp}=0
$$

At the availability of initial data at grid points or at the points scattered over the space it is very convenient to map the invariant characteristics and perform an analysis of the fields and its correlation, and regional distribution depending on the character of variability of the vector value.

It should be emphasized that $\mu$ is not a "correlation coefficient vector of random variables", so we call this value - "index of the vector correlation." Its properties are different from the correlation coefficient of scalar random variables. This index is very useful for express-analysis at the preliminary stage to estimate the distribution in space of level correlation of vector values. In the second stage of express-analysis the properties of the relationship of the vectors are describing by indicators of collinear and orthogonal correlation. We emphasize once again that the indicators of vector correlation $r_{\uparrow \downarrow}$, and $r_{\perp}$ must be analyzed jointly. This two-step approach could be useful used for the analysis of the fields defined on a plurality of points.

\section{Comparison of Model Calculations with Full-Scale Data}

Only satellite observation techniques allow a detailed description of fields of any environmental parameters. In the My Ocean FP7 project the full-scale data $\vec{V}(\vec{r}, t)$ describing the ice drift fields in the Arctic Ocean, derived from satellite information (namely IFREMER ${ }^{(\mathrm{b})}$ and Pathfinder data ${ }^{(\mathrm{d})}$ ), were used for full quantitative and qualitative comparison of the TOPAZ model calculations ${ }^{(\mathrm{e})} \vec{U}(\vec{r}, t)$ using the correlation indicators $\mu, r_{\uparrow \downarrow}$ and $r_{\perp}$.

Here as an example, illustrating of using the vectorial-algebraic approach for validation, we shall consider the spatial distributions of the correlation indexes $r_{\uparrow \downarrow}, r_{\perp}$ and $\mu$, giving information on consistency of the drift fields. For this example, the TOPAZ drift fields and IFREMER data for winter 2006-2007 were used. For demonstration the method, 

Data Using a Vector Correlation Indexes in an Invariant Form

it was important to choose a period that the difference between the model estimates and the reference (observation) data were noticeable. Recall that in 2007 was registered a minimum area of old ice and sufficiently high dynamics of the ice cover (later more significant minimum was observed in 2012).

Mean ice drift fields as well as for TOPAZ and IFREMER $\left(\vec{m}_{\vec{V}}\right)$ are presented on Fig. 2. Really it is a typical large scale space structure of ice drift in the Arctic Ocean including a large anticyclonic gyre in the Amerasian subbasin and Transarctic current is going from the Chukchi, East-Siberian and Laptev Seas via North Pole region to the Fram Strait. One will notice that the drift patterns in the TOPAZ model results are simplified and smoother than in observations. In particular, the model shows too strong ice drift north of the Canadian Archipelago while the observations demonstrate incoherent and rather slow drift.

For the initial time series of the drift at some grid points with an space interval of $125 \mathrm{~km}$ the indexes $\mu$, $r_{\uparrow \downarrow}$ and $r_{\perp}$ were calculated and charts were constructed (Fig. 3) as well as for the TOPAZ free run realization VS IFREMER data and the TOPAZ reanalysis fields VS IFREMER data. It is appropriate to remind that in this case we compare not simply the average initial fields, but the degree of consistency of temporal and spatial variability of the TOPAZ and IFREMER drift fields in winter 2006-2007 while the index values at each field point are calculated from the time series at each specific point of the computational grid.

So as can be seen from the charts presented, the total relation between the TOPAZ and IFREMER fields (Fig. 3a) exists sufficiently high in general for both TOPAZ realizations. Nevertheless for the free run data this relation is less and more heterogeneous in space. In particular, the correlation is decreasing in the coastal zones: both close to North of the Canadian Archipelago and in the marginal seas (the Chukchi Sea, East Siberian Sea and Beaufort Sea), Analysis of the collinear correlation index values and the field distribution (Fig. 3b) demonstrates interdependence of the TOPAZ and IFREMER drift characteristics mainly via coherent variations of drift magnitudes. On the contrary, the contribution of coordinated changes of directions of the drift vectors, characterised by $r_{\perp}$, into the total correlation index has no essential significance in most regions (Fig. 3c).

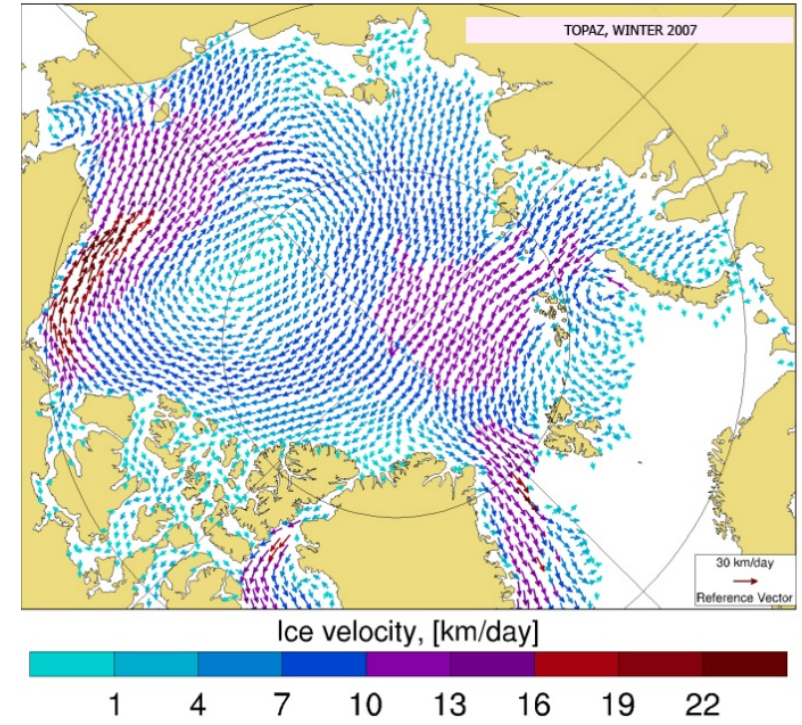

(a) Based on TOPAZ (free-run) data

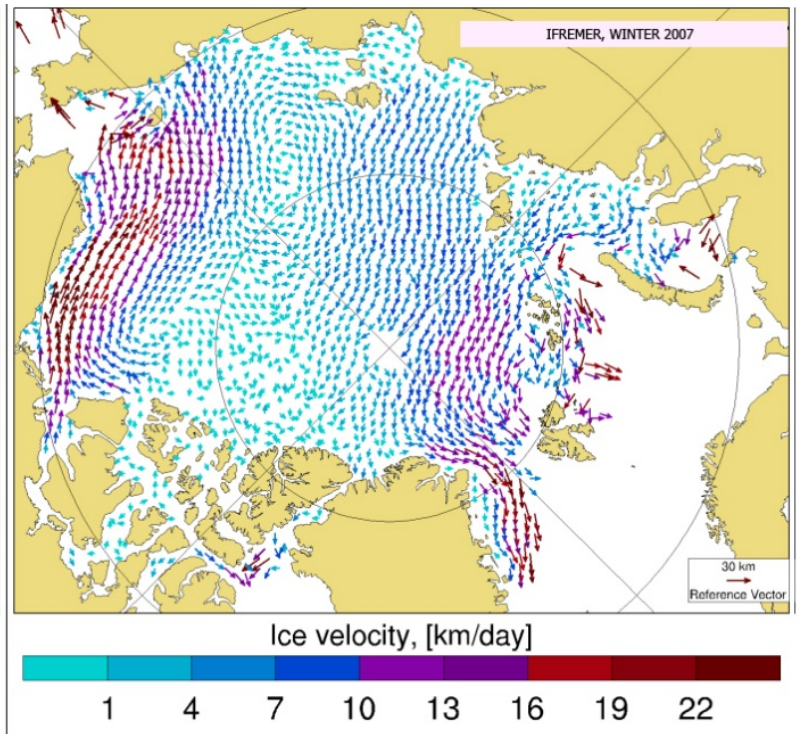

(b) Based on IFREMER data

Fig. 2 Large-scale spatial structure of sea ice drift field in the Arctic Ocean (fields of the vectorial mathematical expectation), winter 2006-2007. 

Data Using a Vector Correlation Indexes in an Invariant Form

(a) Indicator of correlation, $\mu$
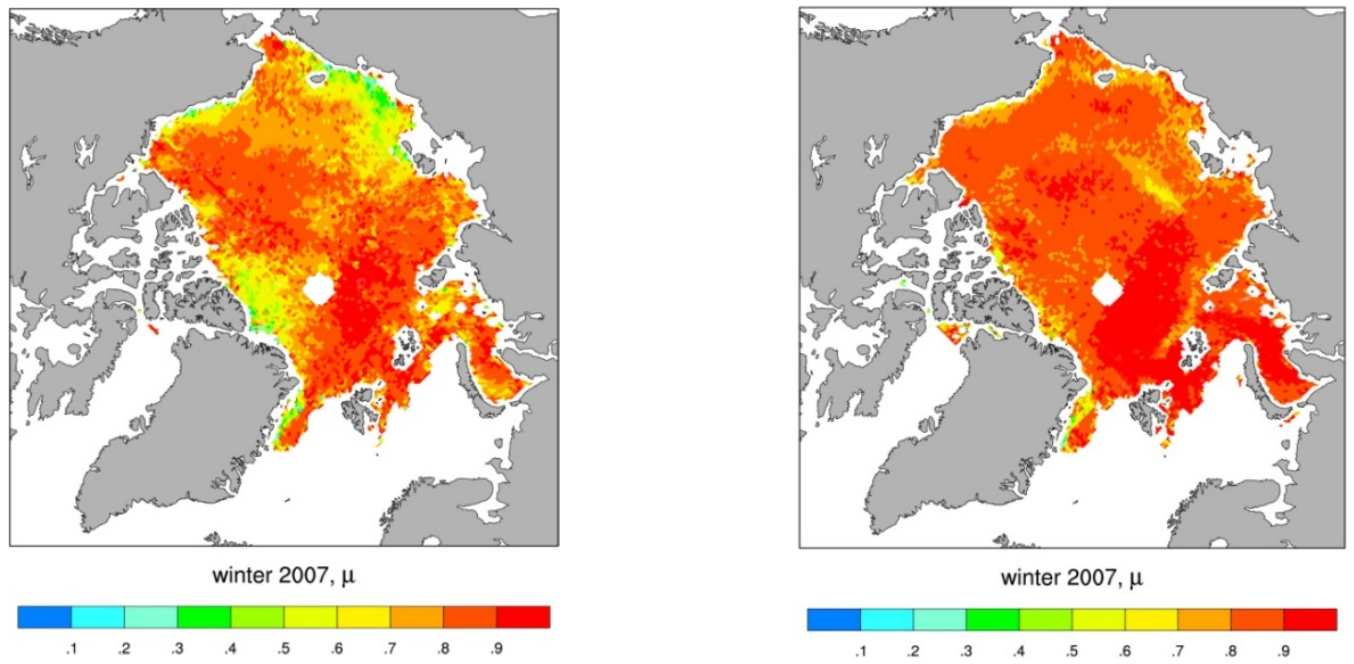

(b) Index of collinear correlation, $\mathrm{r}_{\uparrow \downarrow}$

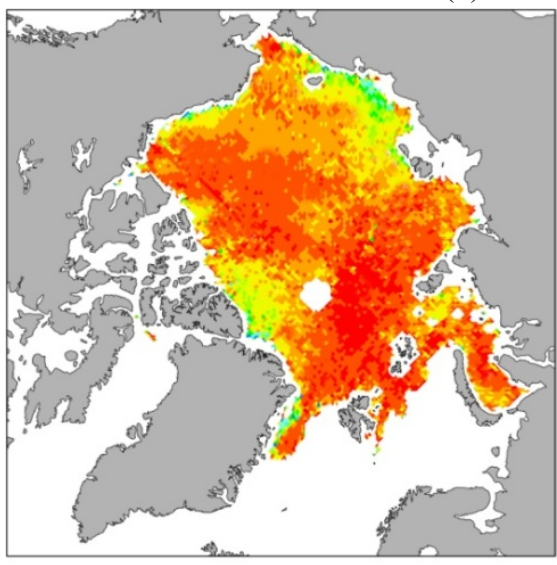

winter 2007, r $\uparrow \downarrow$

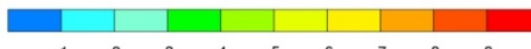

(c) Index of orthogonal correlation, $r_{\perp}$

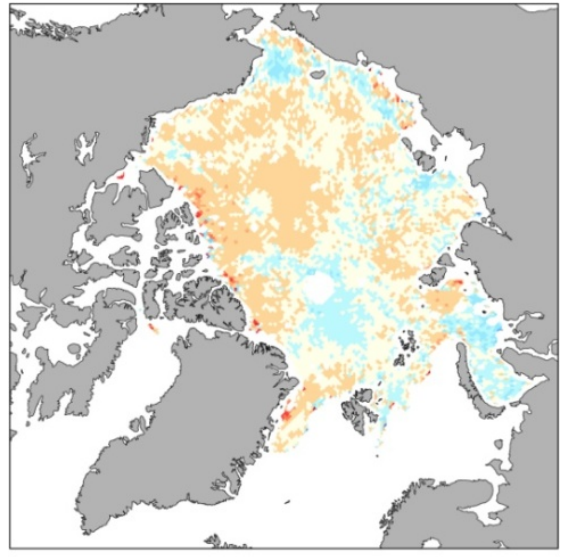

winter 2007, $\mathrm{r} \perp$

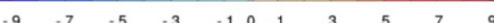

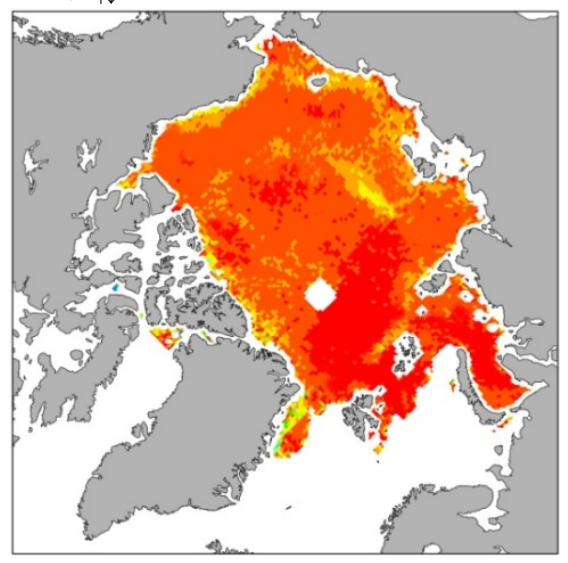

winter 2007, r $\uparrow$
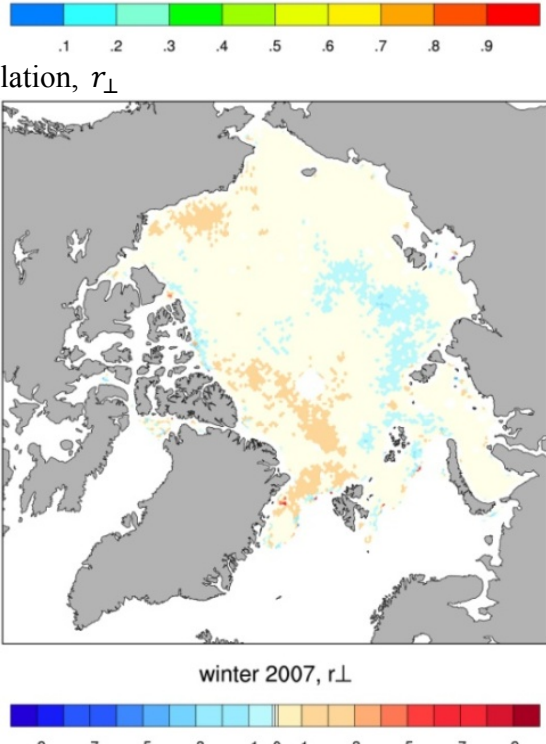

Fig. 3 Correlation indexes for TOPAZ and IFREMER based sea ice drift fields, winter 2006-2007. LEFT: TOPAZ (free-run) VSIFREMER based data set, RIGHT: TOPAZ (reanalysis) VS IFREMER based data set. 

Data Using a Vector Correlation Indexes in an Invariant Form

(a) Linear invariant (total variability), $I_{1}$
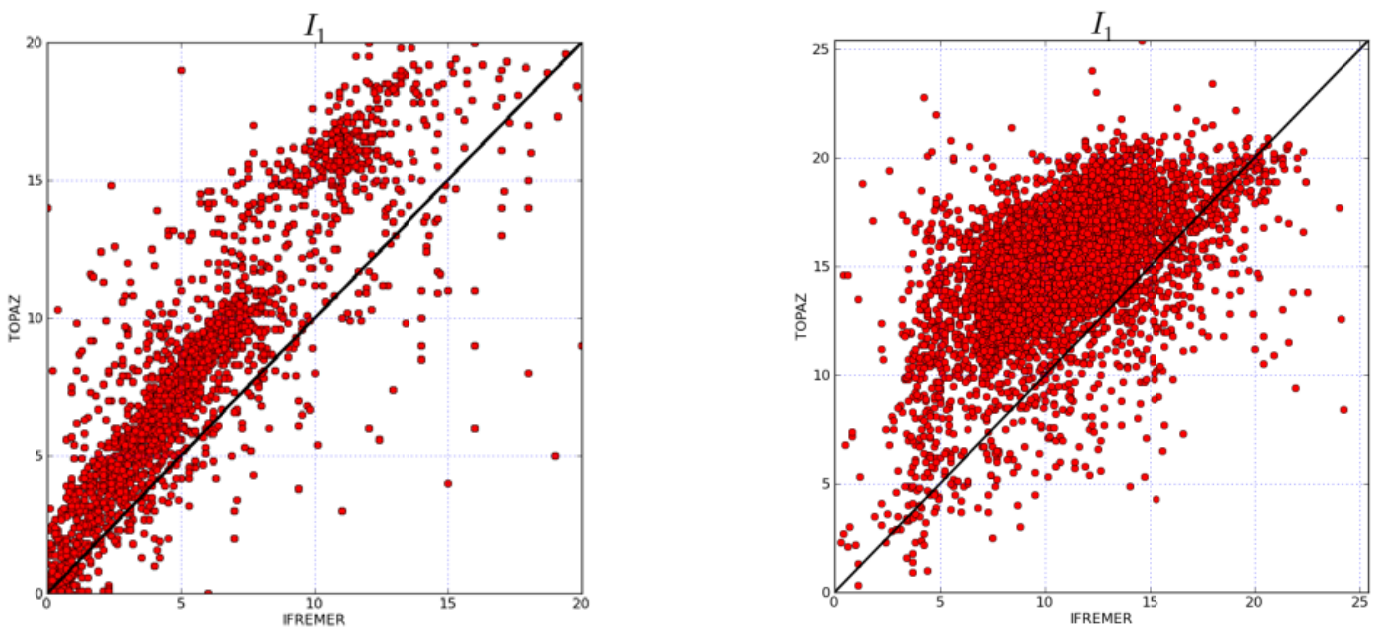

(b) Semi-major axis, $\lambda_{1}$
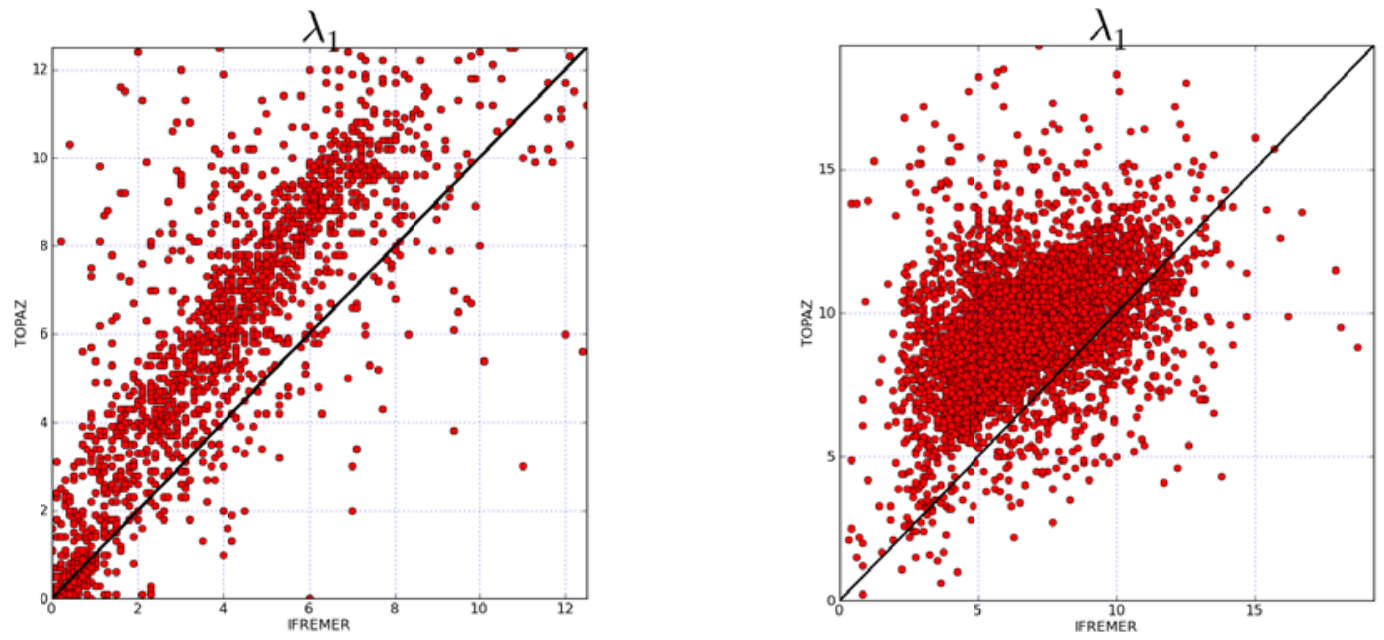

(c) Semi-minor axis, $\lambda_{2}$
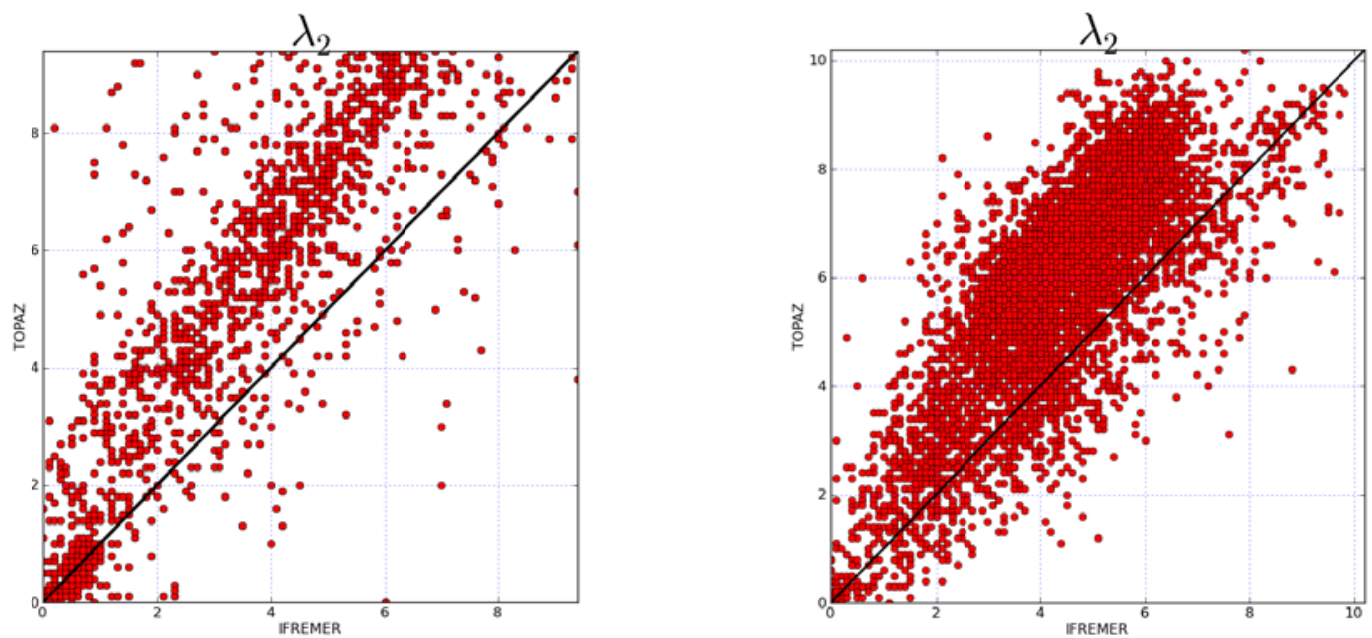

Fig. 4 Comparison of some invariant characteristics for TOPAZ and IFREMER based sea ice drift fields, winter 2006-2007. Source: LEFT:TOPAZ (free-run) VSIFREMER based data set; RIGHT: TOPAZ (reanalysis) VS IFREMER based data set. TOPAZ (ordinate axis), IFREMER based data (abscissa axis). 
Magnitude
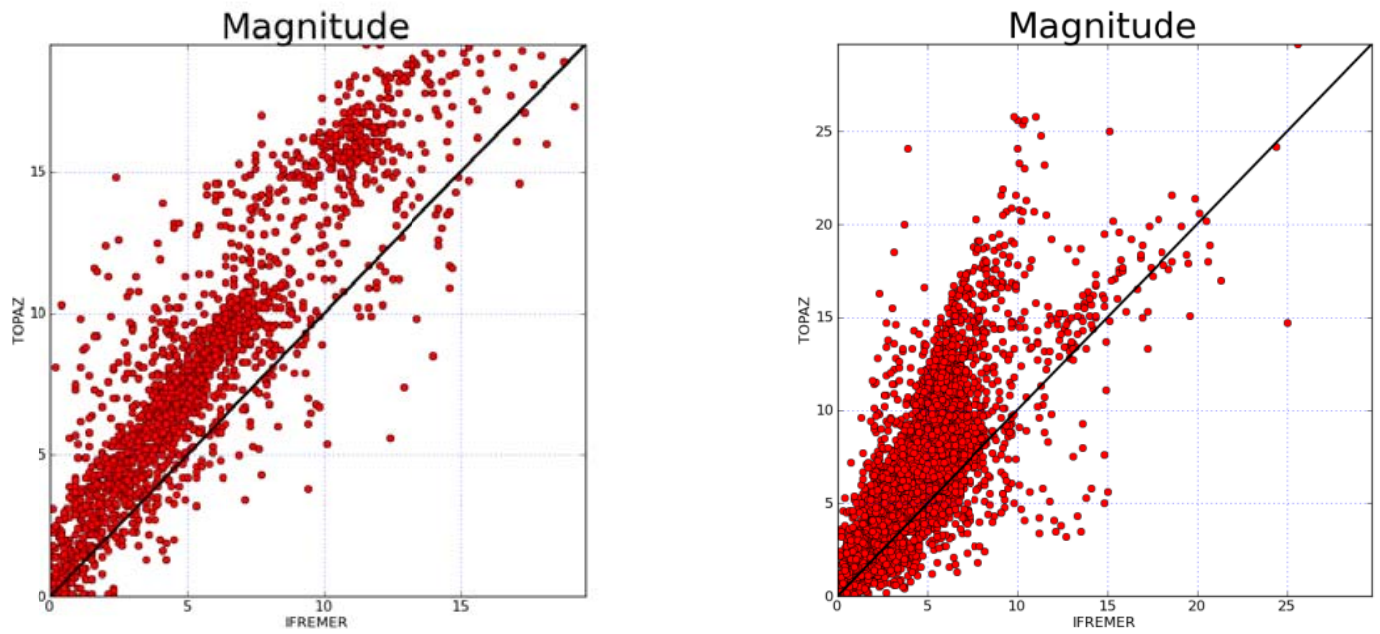

Fig. 5 Comparison of magnitudes for the ice drift fields of the TOPAZ free run and TOPAZ reanalysis results (ordinate axis) with IFREMER based data set (abscissa axis), winter 2006-2007.

Comparing results of TOPAZ (free-run) VS IFREMER based data and TOPAZ (reanalysis) VS IFREMER based data, it is possible to see that in the case of "reanalysis" version the fields of all correlation indexes are more homogeneous, and values of $\mu, r_{\uparrow \downarrow}$ are higher, but $r_{\perp}$ comes practically to zero. It is understandable because the satellite data were assimilated into TOPAZ data. Zones with lower correlation values remain in the same areas and are confined to the continental slope North of the Canadian Archipelago, to North from Barents Sea and Novosibirskiye (New Siberian) Islands, and shallow waters of the marginal seas - the Beaufort, Chukchi Sea, East Siberian and Laptev Seas.

In Fig. 4a diagrams of invariant characteristics for the ice drift fields of the TOPAZ (free run) and TOPAZ (reanalysis) results - ordinate axis, with IFREMER data set-abscissa axis-for winter 2006-2007 are presented. As can be seen as well as linear invariant $I_{1}$, semi-major and minor axis- $\lambda_{1}, \lambda_{2}$ for TOPAZ (free-run) data characterizing the total dispersion and dispersion along the axis $\lambda_{1}, \lambda_{2}$, are systematically overestimated. This overestimation remains also for reanalysis and is consistent with the results from Ref. [7], pointing towards a general deficiency of the EVP model. If we evaluate only the magnitude (Fig. 5), we arrive at the same result — the model estimates the drift velocity generally overestimated.

It should be emphasized that the overestimation module drift velocity vector does not affect the value of the correlation index, as the condition in Eq. (16) is only necessary but it is not sufficient. One of the causes of this effect can be connected with the fact that covariation is a central moment of the distribution of probabilities, i.e., it determines closeness of dependence between the values centered to the mathematical expectation. For example, if the model simulates ideally the vector direction $\varphi_{\vec{U}}=\varphi_{\vec{V}}$ and overestimates (or underestimates) the module by a constant value $U=V \pm$ const, then the condition in Eq. (16) will be fulfilled all the same. This means that the rapid analysis of communication fields (or rows) of vector quantities within the vector-algebraic method must also involve information about the values of invariant characteristics.

It should be noted, the excess value of the drift velocity model can lead to erroneous calculation estimates the removal of ice outside the Arctic Basin and underestimation in the calculation of the thickness of the ice through which, in turn, can lead to 
inaccurate estimates of climate variability ice cover.

It should be noted that approximately the same regularities were detected during evaluation of the My Ocean Project by using IFREMER data for other time periods, and also by using Pathfinder data ${ }^{(\mathrm{d})}$.

\section{Conclusions}

Summarizing this analysis one can conclude that TOPAZ drift model is adequate describing the structure and time variability of drift fields, but overestimates the magnitudes and dispersion of ice drift. Such results can be interpreted as a known deficiency of the EVP ice rheology used in the model. The reason for lower correlation near coast could be both rheology and thermodynamical issues as the biases of the free run reduce the overlap between observations and model data. It needs to introduce corrections to the model parameters.

This article demonstrates also the advantages of the vectorial-algebraic approach for the analysis of vector series where a Euclidean vector is used as a model, and the vector temporal processes are described by a small set of scalar invariant characteristics, derived from the dispersion tensors. The method allows more rigorous mathematics and most adequately describe vector processes, construct the fields of these values (if the corresponding data are available), perform an inter-comparison of the uniform series or the fields with a different time interval, or model and observed values. This approach could be used also for describing of the relationship of the fields of wind or currents, etc. The method has a large potential for describing the temporal variability of the vector series and the fields at different scales, for zonation of regions by means of different criteria of temporal variability or by the characteristics of relationship of the vector time series and the fields.

\section{Acknowledgements}

The authors are grateful to Prof. Valentine A. Rozkov (St. Petersburg State University) for valuable advice given in the process of manuscript preparation, as well as to members of the Oceanographic committee of the Russian Geographical Society for an efficient discussion during a seminar, Dr. Laurent Bertino for the provision of the model data for testing, Dr. François Counillon has prepared the TOPAZ4 model output for the present analysis.

The work was carried out in the framework of WP5 of the My Ocean FP7 Project (2009-2011) and My Ocean 2 Project (2011-2014).

\section{References}

[1] Belyshev, A. P., Klevantsov, Y. P., and Rozhkov, V. A. 1983. "Probabilistic Analysis of Sea Currents." Russian. 264.

[2] Belyshev, A. P., Klevantsov, Y. P., and Rozhkov, V. A. 1981. "On Illusions and Reality in the Methods of Analysis of Sea Currents." In Proc. of the State Oceanographic Institute 157: 3-19.

[3] Crosby, D. S., Breaker, L. C., and Gemmill, W. H. 1993. "A Proposed Definition for Vector Correlation in Geophysics: Theory and Application." Journal of Atmospheric and Oceanic Technology 10: 355-67.

[4] Sakov, P., Counillon, F., Bertino, L., Lisæter, K. A., Oke, P. R., and Korablev, A. 2012. "TOPAZ4: An Ocean-Sea Ice Data Assimilation System for the North Atlantic and Arctic." Ocean Science 8: 633-56.

[5] Bleck, R. 2002. "An Oceanic General Circulation Model Framed in Hybrid Isopycnic-Cartesian Coordinates." Ocean. Mod. 4: 55-88.

[6] Lisaeter, K., Rosanova, J., and Evensen, G. 2003. "Assimilation of Ice Concentration in a Coupled Ice-Ocean Model, Using the Ensemble Kalman Filter." Ocean Dyn. 53: 368-88.

[7] Girard, L., Weiss, J., Molines, J. M., Barnier, B., and Bouillon, S. 2009. "Evaluation of High-Resolution Sea Ice Models on the Basis of Statistical and Scaling Properties of Arctic Sea Ice Drift and Deformation." Journal of Geophysical Research: Oceans 114 (C8).

[8] Kochin, N. E. 1961. "Vector Analysis and Beginning of Tensor Calculation." M., Publishing House of the USSR Academy of Science 425.

[9] Obukhov, A. M. 1945. "Theory of Vector Correlation." Scientific Notes of Moscow State University 45: 56-71.

[10] Klevantsov, Y. P., Mikulinskaya, S. M., and Rozhkov, V. A. 1996. "On the Analysis of Sea Current Speed Vectors." Meteorology and Hydrology 9: 96-105.

[11] Gudkovich, Z. M. 1965. "Correlation Method of Processing of Observations of Currents, 1965." Problems 
Validation of the Model Obtained Ice Drift Fields Based on Satellite Derived

Data Using a Vector Correlation Indexes in an Invariant Form

of the Arctic and the Antarctic 21: 56-60.

[12] Ivanov, N. E. 2004. "On the Characteristics of Correlation of Wind Speeds, Sea Currents and Ice Drift." Meteorology and Hydrology 8: 61-72. 